\title{
Admissible functions for the Dirichlet space
}

by

\section{Javad Mashreghi (Québec) and Mahmood Shabankhah (Marseille)}

Abstract. Zero sets and uniqueness sets of the classical Dirichlet space $\mathcal{D}$ are not completely characterized yet. We define the concept of admissible functions for the Dirichlet space and then apply them to obtain a new class of zero sets for $\mathcal{D}$. Then we discuss the relation between the zero sets of $\mathcal{D}$ and those of $\mathcal{A}^{\infty}$.

1. Introduction. Let $f(z)=\sum_{n=0}^{\infty} a_{n} z^{n}$ be holomorphic on the open unit disk $\mathbb{D}$. Then, by direct verification, we obtain

$$
\mathcal{D}(f):=\frac{1}{\pi} \int_{\mathbb{D}}\left|f^{\prime}(z)\right|^{2} d A(z)=\sum_{n=1}^{\infty} n\left|a_{n}\right|^{2},
$$

where $d A$ is the two-dimensional Lebesgue measure. The Dirichlet space is

$$
\mathcal{D}:=\{f \in \operatorname{Hol}(\mathbb{D}): \mathcal{D}(f)<\infty\} .
$$

It is clear that the classical Hardy space $H^{2}(\mathbb{D})$ contains the Dirichlet space $\mathcal{D}$ as a proper subclass [M, p. 104]. With the norm

$$
\|f\|_{\mathcal{D}}^{2}=\mathcal{D}(f)+\|f\|_{H^{2}}^{2},
$$

the Dirichlet space becomes a Hilbert space of analytic functions on the open unit disc whose inner product is given by

$$
\langle f, g\rangle=\sum_{n=0}^{\infty}(n+1) a_{n} \bar{b}_{n},
$$

where $f(z)=\sum_{n=0}^{\infty} a_{n} z^{n}$ and $g(z)=\sum_{n=0}^{\infty} b_{n} z^{n}$ are arbitrary elements of $\mathcal{D}$.

A sequence $\left(z_{n}\right)_{n \geq 1}$ in $\mathbb{D}$ is called a zero set for $\mathcal{D}$ provided that there is an element $f \in \mathcal{D}, f \not \equiv 0$, such that $f\left(z_{n}\right)=0, n \geq 1$. Since $\mathcal{D} \subset H^{2}(\mathbb{D})$, it is well-known that a necessary condition for a zero set is the Blaschke condition

$$
\sum_{n=1}^{\infty}\left(1-\left|z_{n}\right|\right)<\infty
$$

2010 Mathematics Subject Classification: Primary 30C15; Secondary 30J10, 30H10, 31C25. Key words and phrases: zero sets, admissible functions, Dirichlet space, Blaschke products. 
The complete characterization of the zero sets of $\mathcal{D}$ is still an open question. Carleson Car2 took the first step in this direction and showed that if a sequence $\left(z_{n}\right)_{n \geq 1}$ in $\mathbb{D}$ satisfies

$$
\sum_{n=1}^{\infty}\left(\frac{1}{-\log \left(1-\left|z_{n}\right|\right)}\right)^{1-\varepsilon}<\infty
$$

for some $\varepsilon>0$, then $\left(z_{n}\right)_{n \geq 1}$ is a zero set for $\mathcal{D}$. Then Shapiro-Shields [SS] improved this result by showing that

$$
\sum_{n=1}^{\infty} \frac{1}{-\log \left(1-\left|z_{n}\right|\right)}<\infty
$$

is enough to ensure that $\left(z_{n}\right)_{n \geq 1}$ is a zero set for $\mathcal{D}$. This is an important generalization of Carleson's result, and in a sense, the best known result up to now. This line of research has been continued by Bogdan [B], Caughran Cau1, Nagel-Rudin-Shapiro [NRS, and Richter-Ross-Sundberg [RRS].

In $\mathrm{MS}$, among other things, we showed that if a Blaschke sequence $\left(z_{n}\right)_{n \geq 1}$ tends to the point 1 and satisfies

$$
\sum_{n=1}^{\infty} \exp \left(-\frac{\beta}{d_{n}^{\alpha}}\right)<\infty
$$

for some $0<\alpha<1$ and $\beta>0$, or

$$
\sum_{n=1}^{\infty} \exp \left(-\frac{\beta}{d_{n} \log \frac{1}{d_{n}} \log _{2} \frac{1}{d_{n}} \cdots \log _{k}^{1+\varepsilon} \frac{1}{d_{n}}}\right)<\infty
$$

for some $\beta>0, \varepsilon>0$, and integer $k \geq 1$, where $d_{n}=\left|1-z_{n}\right|$, then $\left(z_{n}\right)_{n \geq 1}$ is a zero set for $\mathcal{D}$. We also slightly generalized the constructive method of Carleson, given in Cau1, to create a uniqueness set. In this paper, we take further steps in this direction. In Section 2, we define the concept of $E$-admissible functions, where $E$ is a Carleson subset of $\mathbb{T}$. Then we give some sufficient conditions to create these functions. This concept is designed to obtain zero sets of the Dirichlet space. In the last section, which is also of independent interest, we generalize a result of Nelson on Carleson sets and then apply it to show that a special class of zero sets of $\mathcal{D}$ and $\mathcal{A}^{\infty}$ coincide. We recall that $\mathcal{A}^{k}=\left\{g \in \operatorname{Hol}(\mathbb{D}): g^{(k)}\right.$ extends continuously to $\left.\overline{\mathbb{D}}\right\}$ and $\mathcal{A}^{\infty}=\bigcap_{k} \mathcal{A}^{k}$.

2. E-admissible functions. In this section, we shall prove a theorem which provides a fairly large class of zero sets for the Dirichlet space. In what follows, Carleson sets will play a prominent role. A closed subset $E$ of the unit circle $\mathbb{T}$ is called a Carleson set if

$$
\int_{\mathbb{T}} \log \operatorname{dist}(\zeta, E)|d \zeta|>-\infty
$$


where dist stands for the Euclidean distance. It is not difficult to show that (2.1) is equivalent to $|E|=0$ and

$$
\sum_{n}\left|I_{n}\right| \log \left|I_{n}\right|>-\infty
$$

where $I_{n}, n \geq 1$, are the complementary open intervals of $E$. Let

$$
E_{t}=\{\zeta \in \mathbb{T}: d(\zeta, E) \leq t\}
$$

where $d$ denotes the arc length on the unit circle. It is shown in Car1 that a closed set $E$ is a Carleson set if and only if

$$
\int_{0}^{\left|E_{t}\right|} \frac{1}{t} d t<\infty
$$

It follows that a finite union of Carleson sets is itself a Carleson set.

The following result gives sufficient conditions for a Blaschke sequence to be a zero set for the Dirichlet space. Note that if $E$ is a Carleson set, then, by [TW, Theorem 1.1], there is a function $f \in \mathcal{A}^{\infty}=\left\{f: f^{(n)} \in\right.$ $\left.H^{\infty}(\mathbb{D}), n \geq 0\right\}$ which vanishes on $E$. Hence, in particular, $f$ satisfies

$$
|f(\zeta)| \leq C \operatorname{dist}(\zeta, E) \quad(\zeta \in \mathbb{T})
$$

See also [Car1, Theorem 1] for a similar but weaker result.

TheOrem 2.1. Let $E$ be a closed subset of $\mathbb{T}$, and let $\varphi:[0,2] \rightarrow[0, \infty)$ be an increasing function such that

$$
S(E, \varphi)=\int_{\mathbb{T}} \frac{\varphi(a \operatorname{dist}(\zeta, E))}{\operatorname{dist}^{2}(\zeta, E)}|d \zeta|<\infty
$$

for some $0<a<1$. Suppose that there exists a non-zero $f \in \mathcal{D}$ such that

$$
|f(\zeta)|^{2} \leq \varphi(a \operatorname{dist}(\zeta, E)) \quad(\text { a.e. on } \mathbb{T}) .
$$

Then $E$ is a Carleson set and every Blaschke sequence $\left(z_{n}\right)_{n \geq 1}$ satisfying

$$
\sum_{n=1}^{\infty} \varphi\left(\operatorname{dist}\left(z_{n}, E\right)\right)<\infty
$$

is a zero set for $\mathcal{D}$.

Proof. By assumption,

$$
\int_{\mathbb{T}} \log \left(\frac{|f(\zeta)|^{2}}{\operatorname{dist}^{2}(\zeta, E)}\right)|d \zeta| \leq \int_{\mathbb{T}} \log \left(\frac{\varphi(a \operatorname{dist}(\zeta, E))}{\operatorname{dist}^{2}(\zeta, E)}\right)|d \zeta|<\infty
$$

and, by a well-known result of F. Riesz,

$$
\int_{\mathbb{T}} \log |f(\zeta)||d \zeta|>-\infty
$$


Hence, we have

$$
\int_{\mathbb{T}} \log \operatorname{dist}(\zeta, E)|d \zeta|>-\infty .
$$

Consequently, $E$ is a Carleson set.

Fix $z \in \mathbb{D}$ and set

$$
E_{z}=\{\zeta \in \mathbb{T}: \operatorname{dist}(\zeta, E) \geq \operatorname{dist}(z, E) / a\} .
$$

Let $\zeta_{0}$ be the closest point of $E$ to $z$. Then, for each $\zeta \in E_{z}$,

$$
\begin{aligned}
|\zeta-z| & \geq\left|\zeta-\zeta_{0}\right|-\left|\zeta_{0}-z\right|=\left|\zeta-\zeta_{0}\right|-\operatorname{dist}(z, E) \\
& \geq \operatorname{dist}(\zeta, E)-\operatorname{dist}(z, E) \geq(1-a) \operatorname{dist}(\zeta, E) .
\end{aligned}
$$

Hence,

$$
\int_{E_{z}} \frac{|f(\zeta)|^{2}}{|\zeta-z|^{2}}|d \zeta| \leq \int_{E_{z}} \frac{\varphi(a \operatorname{dist}(\zeta, E))}{|\zeta-z|^{2}}|d \zeta| \leq\left(\frac{1}{1-a}\right)^{2} S(E, \varphi) .
$$

On the other hand, on $\mathbb{T} \backslash E_{z}$, we have

$$
\begin{aligned}
\int_{\mathbb{T} \backslash E_{z}} \frac{|f(\zeta)|^{2}}{|\zeta-z|^{2}}|d \zeta| & \leq \int_{\mathbb{T} \backslash E_{z}} \frac{\varphi(a \operatorname{dist}(\zeta, E))}{|\zeta-z|^{2}}|d \zeta| \\
& \leq \varphi(\operatorname{dist}(z, E)) \int_{\mathbb{T} \backslash E_{z}} \frac{|d \zeta|}{|\zeta-z|^{2}} \leq \frac{2 \pi}{1-|z|^{2}} \varphi(\operatorname{dist}(z, E)) .
\end{aligned}
$$

So, for every $z \in \mathbb{D}$,

$$
\left(1-|z|^{2}\right) \int_{\mathbb{T}}\left|\frac{f(\zeta)}{\zeta-z}\right|^{2}|d \zeta| \leq\left(\frac{1}{1-a}\right)^{2} S(E, \varphi)\left(1-|z|^{2}\right)+2 \pi \varphi(\operatorname{dist}(z, E)) .
$$

Therefore, by our assumption,

$$
\sum_{n=1}^{\infty}\left(1-\left|z_{n}\right|^{2}\right) \int_{\mathbb{T}}\left|\frac{f(\zeta)}{\zeta-z_{n}}\right|^{2}|d \zeta|<\infty .
$$

Let $B$ be the Blaschke product formed by $\left(z_{n}\right)_{n \geq 1}$. It follows from Carleson's formula Car3 that

$$
\mathcal{D}(B f)=\mathcal{D}(f)+\sum_{n=1}^{\infty}\left(1-\left|z_{n}\right|^{2}\right) \int_{\mathbb{T}}\left|\frac{f(\zeta)}{\zeta-z_{n}}\right|^{2}|d \zeta|<\infty .
$$

Thus, $\left(z_{n}\right)_{n \geq 1}$ is a zero set for the Dirichlet space.

An easy assumption which ensures $S(E, \varphi)<\infty$ is that $\varphi(t) \leq C t^{2}$, $C>0$, which is fulfilled by all functions we consider in applications. But the existence of $f$ is more delicate and needs further considerations. Theorem 2.1 suggests the following definition. For a Carleson set $E$, we define 
an increasing function $\varphi:[0,2] \rightarrow[0, \infty)$ to be $E$-admissible if $S(E, \varphi)<\infty$ and there is a non-zero function $f \in \mathcal{D}$ such that

$$
|f(\zeta)|^{2} \leq \varphi(a \operatorname{dist}(\zeta, E)) \quad(\text { a.e. on } \mathbb{T})
$$

for some $a \in(0,1)$. With this new terminology, the assumptions of Theorem 2.1 simply mean that $\varphi$ is $E$-admissible. Moreover, (1.1) and 1.2 both become special cases of this result, and we can say that the functions

$$
\varphi(t)=\exp \left(-\frac{\beta}{t^{\alpha}}\right)
$$

where $0<\alpha<1, \beta>0$, and

$$
\varphi(t)=\exp \left(-\frac{\beta}{t \log \frac{1}{t} \log _{2} \frac{1}{t} \cdots \log _{k}^{1+\varepsilon} \frac{1}{t}}\right),
$$

where $\beta>0, \varepsilon>0$, and $k \geq 1$, are $\{1\}$-admissible.

The key step in applying Theorem 2.1 is to find a proper admissible function. The following result is an attempt to give sufficient conditions under which such a function always exists. A prototype of the function $\varphi$ which is considered below is $\varphi(t)=t^{\alpha}, \alpha \geq 2$.

Proposition 2.2. Let $\varphi:[0,2] \rightarrow[0, \infty)$ be an increasing continuously differentiable function with $\varphi(0)=0, \varphi(t)>0$ for $t>0$, and such that $t \varphi^{\prime}(t) / \varphi(t)$ is increasing, and $\varphi^{\prime}(t) / t$ is bounded on $(0,2]$. Then $\varphi$ is $E$ admissible for every Carleson set $E$.

Proof. Since $\varphi^{\prime}(t) / t$ is bounded, we have $\varphi(0)=\varphi^{\prime}(0)=0$ and also $\varphi(t) \leq C t^{2}$. Hence, $S(E, \varphi)<\infty$. Since $E$ is a Carleson set, by a wellknown result of Carleson Car1, there is an outer function $g \in \mathcal{A}^{1}(\mathbb{D})$ which vanishes on $E$. In particular, we have

$$
|g(\zeta)| \leq C \operatorname{dist}(\zeta, E) \quad(\zeta \in \mathbb{T}) .
$$

Multiplying $g$ by a proper constant, without loss of generality, we may assume that $C=a \in(0,1)$. Hence,

$$
\varphi(|g(\zeta)|) \leq \varphi(a \operatorname{dist}(\zeta, E)) \quad(\zeta \in \mathbb{T}) .
$$

Since $t \varphi^{\prime}(t) / \varphi(t)$ and $\varphi(t) \varphi^{\prime}(t) / t$ are both bounded on $(0,2]$, it follows that, for almost every $\zeta_{1}, \zeta_{2} \in \mathbb{T}$,

$$
\begin{aligned}
\left|\log \varphi\left(\left|g\left(\zeta_{1}\right)\right|\right)-\log \varphi\left(\left|g\left(\zeta_{2}\right)\right|\right)\right| & \leq C|\log | g\left(\zeta_{1}\right)|-\log | g\left(\zeta_{2}\right)||, \\
\left|\varphi\left(\left|g\left(\zeta_{1}\right)\right|\right)-\varphi\left(\left|g\left(\zeta_{2}\right)\right|\right)\right| & \leq\left. C|| g\left(\zeta_{1}\right)\right|^{2}-\left|g\left(\zeta_{2}\right)\right|^{2} \mid,
\end{aligned}
$$

where $C$ is a positive constant. On the other hand, since $t \varphi^{\prime}(t) / \varphi(t)$ is increasing, $\log \varphi(t)$ is a convex function of $\log t$. Hence, Jensen's inequality 
together with the fact that $\log |g|$ is integrable implies that

$$
\frac{1}{2 \pi} \int_{\mathbb{T}} \log \varphi(|g(\zeta)|)|d \zeta| \geq \log \varphi\left(\exp \left\{\frac{1}{2 \pi} \int_{\mathbb{T}} \log |g(\zeta)||d \zeta|\right\}\right)>-\infty .
$$

Let $f$ be the outer function such that $|f(\zeta)|^{2}=\varphi(|g(\zeta)|), \zeta \in \mathbb{T}$. Clearly, $f \in H^{2}(\mathbb{T})$. Furthermore, by Carleson's formula Car3 and by 2.5 and (2.6),

$$
\begin{aligned}
\mathcal{D}(f) & =\iint_{\mathbb{T}} \frac{\left(\log \left|f\left(\zeta_{1}\right)\right|-\log \left|f\left(\zeta_{2}\right)\right|\right)\left(\left|f\left(\zeta_{1}\right)\right|^{2}-\left|f\left(\zeta_{2}\right)\right|^{2}\right)}{\left|\zeta_{1}-\zeta_{2}\right|^{2}} \frac{\left|d \zeta_{1}\right|\left|d \zeta_{2}\right|}{4 \pi^{2}} \\
& \leq C \mathcal{D}(g)<\infty .
\end{aligned}
$$

Therefore, $f \in \mathcal{D}$.

Since Proposition 2.2 gives us an $E$-admissible function for any Carleson set $E$, we can apply Theorem 2.1 to get a more practical way to obtain zero sets for $\mathcal{D}$. More precisely, every Blaschke sequence $\left(z_{n}\right)_{n \geq 1}$ satisfying

$$
\sum_{n=1}^{\infty} \varphi\left(\operatorname{dist}\left(z_{n}, E\right)\right)<\infty
$$

where $E$ is a Carleson set and $\varphi$ fulfills the conditions of Proposition 2.2, is a zero set for $\mathcal{D}$. In particular, if a Blaschke sequence $\left(z_{n}\right)_{n \geq 1}$ is such that

$$
\sum_{n=1}^{\infty} \operatorname{dist}\left(z_{n}, E\right)^{\alpha}<\infty
$$

for some $\alpha>0$, then $\left(z_{n}\right)_{n \geq 1}$ is a zero set for $\mathcal{D}$. A partial converse to this last result will be discussed in the next section (Theorem 3.2).

Let us make a trivial, but useful, remark about the condition (2.7). For a fixed $\alpha>0$, let $\mathcal{Z}_{\alpha}$ denote the family of all sequences which satisfy (2.7). Then the chain $\left(\mathcal{Z}_{\alpha}\right)_{\alpha>0}$ is increasing, i.e.

$$
\alpha \leq \beta \Rightarrow \mathcal{Z}_{\alpha} \subset \mathcal{Z}_{\beta} .
$$

Therefore, if (2.7) holds for some $\alpha$, then it certainly holds for all $\beta \geq \alpha$, and thus, without loss of generality, we can assume that $\alpha \geq 2$ and then apply Proposition 2.2. Generally speaking, to prove any result whose hypothesis is (2.7) with $\alpha>\alpha_{0}$, we can assume that $\alpha$ is as large as we wish, and by decreasing the value of $\alpha_{0}$ we do not increase the scope of result.

3. More on Carleson sets. In this section we will show that the property that every Blaschke sequence $\left(z_{n}\right)_{n \geq 1}$ satisfying (2.7), with some $\alpha \geq 1$, be a zero set for $\mathcal{D}$ is characteristic of Carleson sets $E$. We further conjecture that this is true for all values of $\alpha>0$. 
It has already been proved by Nelson that if $E$ is a Carleson set and if $\left(z_{n}\right)_{n \geq 1}$ is a Blaschke sequence satisfying (2.7), then $\left(z_{n}\right)_{n \geq 1}$ is even a zero set for $\mathcal{A}^{\infty}$ [Ne, Theorem 1.3]. So, nothing new could be gained out of Theorem 2.1 if one just considers the case of power functions $\varphi(t)=t^{\alpha}$, $\alpha>0$.

Nelson's proof goes as follows. In the first place, he shows, by using delicate results of Caughran [Cau2, Theorem 2] and Novinger [No, Theorem 4.3], that a Blaschke sequence $\left(r_{n} e^{i \theta_{n}}\right)_{n \geq 1} \subset \mathbb{D}$ is a zero set for $\mathcal{A}^{\infty}$ if and only if $\overline{\left\{e^{i \theta_{n}}: n \geq 1\right\}}$ is a Carleson set [Ne, Theorem 1.2]. Armed with this characterization, Nelson then completes his proof of [Ne, Theorem 1.3] by showing that if 2.7 is satisfied by a Blaschke sequence $\left(r_{n} e^{i \theta_{n}}\right)_{n \geq 1}$, then $\overline{\left\{e^{i \theta_{n}}: n \geq 1\right\}}$ is a Carleson set, and thus it has to be a zero set for $\mathcal{A}^{\infty}$.

The following lemma slightly generalizes this last result of Nelson implicitly proved in the course of the proof of [Ne, Theorem 1.3], where he only considers power functions $\varphi(t)=t^{\alpha}, \alpha \geq 1$. At the same time, our proof is considerably shorter.

Lemma 3.1. Let $\varphi:[0,2] \rightarrow[0, \infty)$, with $\varphi(0)=0$, be a strictly increasing continuously differentiable function such that

(i) $\limsup _{t \rightarrow 0^{+}} \frac{t \varphi^{\prime}(t)}{\varphi(t)}<\infty$

(ii) $\liminf _{t \rightarrow 0^{+}} \frac{\log \varphi(t)}{\log t}>0$.

Let $E$ be a Carleson set and let $\left(e^{i \theta_{n}}\right)_{n \geq 1}$ be a sequence on the unit circle $\mathbb{T}$ such that

$$
\sum_{n=1}^{\infty} \varphi\left(d\left(e^{i \theta_{n}}, E\right)\right)<\infty .
$$

Then $\overline{\left\{e^{i \theta_{n}}: n \geq 1\right\}}$ is also a Carleson set.

Proof. We shall show that $F=E \cup \overline{\left\{e^{i \theta_{n}}: n \geq 1\right\}}$ is a Carleson set. Take the integer $k \geq 1$ such that

$$
\frac{\varphi^{\prime}(t)}{\varphi(t)} \leq \frac{k}{t} \quad \text { and } \quad \varphi^{k}(t) \leq t \quad(t \in(0,2])
$$

Since we can divide and multiply the left side of the first equation by $\varphi^{k-1}$, and the assumptions of the lemma hold clearly for $\varphi^{k}$, without loss of generality, we assume that $k=1$, and $\varphi$ satisfies

$$
\frac{\varphi^{\prime}(t)}{\varphi(t)} \leq \frac{c}{t} \quad \text { and } \quad \varphi(t) \leq t \quad(t \in(0,2])
$$

where $c \geq 1$ is a constant. 
Write $\psi=\varphi^{-1}$. For $t \in(0,1]$, we have

Let

$$
F_{t} \subset E_{2 t} \cup \bigcup_{d\left(e^{\left.i \theta_{n}, E\right)>t}\right.}\left[\theta_{n}-t, \theta_{n}+t\right] \subset E_{2 \psi(t)} \cup \bigcup_{d\left(e^{i \theta_{n}}, E\right)>\psi(t)}\left[\theta_{n}-t, \theta_{n}+t\right] .
$$

$$
N_{t}=\operatorname{Card}\left\{n: \varphi\left(d\left(e^{i \theta_{n}}, E\right)\right)>t\right\}=\operatorname{Card}\left\{n: d\left(e^{i \theta_{n}}, E\right)>\psi(t)\right\} .
$$

Hence, by assumption,

$$
\int_{0}^{\infty} N_{t} d t=\sum_{n=1}^{\infty} \varphi\left(d\left(e^{i \theta_{n}}, E\right)\right)<\infty .
$$

It now follows that

$$
\begin{aligned}
& \int_{0} \frac{\left|F_{t}\right|}{t} d t \leq \int_{0} \frac{\left|E_{2 \psi(t)}\right|}{t} d t+2 \int_{0} N_{t} d t=\int_{0} \frac{\left|E_{2 t}\right|}{\varphi(t)} \varphi^{\prime}(t) d t+2 \int_{0} N_{t} d t \\
& \leq c \int_{0} \frac{\left|E_{2 t}\right|}{t} d t+2 \int_{0} N_{t} d t<\infty .
\end{aligned}
$$

Therefore, $F$ is a Carleson set.

Combined with [Ne, Theorem 1.2], Lemma 3.1 leads to the following generalization of [Ne, Theorem 1.3]: If $\varphi$ and $E$ satisfy the hypotheses of Lemma 3.1, then every Blaschke sequence $\left(r_{n} e^{i \theta_{n}}\right)_{n \geq 1}$ satisfying

$$
\sum_{n=1}^{\infty} \varphi\left(d\left(e^{i \theta_{n}}, E\right)\right)<\infty
$$

is a zero set for $\mathcal{A}^{\infty}$. It is natural to ask whether the assumption on $E$ could be somehow weakened. The following result establishes a partial solution to this problem in the case when $\varphi(t)=t^{\alpha}, \alpha \geq 1$. Note that due to the inequalities

$$
\operatorname{dist}\left(e^{i \theta}, E\right) / 2 \leq \operatorname{dist}\left(r e^{i \theta}, E\right) \leq(1-r)+\operatorname{dist}\left(e^{i \theta}, E\right),
$$

where $r e^{i \theta} \in \mathbb{D}$ and $E$ is a closed subset of $\mathbb{T}$, the assumptions $\sum_{n} \operatorname{dist}\left(z_{n}, E\right)^{\alpha}$ $<\infty$ and $\sum_{n} \operatorname{dist}\left(e^{i \theta_{n}}, E\right)^{\alpha}<\infty$ are interchangeable if $\alpha \geq 1$ and $\left(z_{n}\right)_{n \geq 1}$ is a Blaschke sequence.

We will also need the notion of Blaschke sets $[\mathrm{B}]$. A set $A \subset \mathbb{D}$ is called a Blaschke set for $\mathcal{D}$ if every Blaschke sequence in $A$ is a zero set for $\mathcal{D}$.

TheOrem 3.2. Let $\alpha \geq 1$ and let $E$ be a closed subset of $\mathbb{T}$. Then the following are equivalent:

(i) Every Blaschke sequence $\left(z_{n}\right)_{n \geq 1}$ satisfying

is a zero set for $\mathcal{A}^{\infty}$.

$$
\sum_{n=1}^{\infty} \operatorname{dist}\left(z_{n}, E\right)^{\alpha}<\infty
$$


(ii) Every Blaschke sequence $\left(z_{n}\right)_{n \geq 1}$ satisfying

is a zero set for $\mathcal{D}$.

$$
\sum_{n=1}^{\infty} \operatorname{dist}\left(z_{n}, E\right)^{\alpha}<\infty
$$

(iii) $E$ is a Carleson set.

Proof. The implication (i) $\Rightarrow$ (ii) is trivial. That (iii) $\Rightarrow$ (i) is Nelson's result [Ne, Theorem 1.3]. So it remains to show that (ii) $\Rightarrow$ (iii). To do so, put

$$
S=\left\{r e^{i \theta} \in \mathbb{D}: 0 \leq r<1, e^{i \theta} \in E\right\}
$$

and let $z_{n}, n \geq 1$, be a Blaschke sequence in $S$. Since

$$
\sum_{n=1}^{\infty} \operatorname{dist}\left(z_{n}, E\right)^{\alpha} \leq \sum_{n=1}^{\infty} 1-r_{n}<\infty,
$$

it follows that $\left(z_{n}\right)_{n \geq 1}$ is a zero set for $\mathcal{D}$. Therefore, by definition, $S$ is a Blaschke set for $\mathcal{D}$ and so by Bogdan's theorem [B, Theorem 1],

$$
\int_{\mathbb{T}} \log \operatorname{dist}(\zeta, S)|d \zeta|>-\infty .
$$

In particular, $E=\bar{S} \cap \mathbb{T}$ is a Carleson set.

REMARK 3.3. The equivalence (i) $\Leftrightarrow$ (iii) above is in fact valid for every $\alpha>0$. To see this, just note that zero sets of $\mathcal{A}^{\infty}$ functions can only accumulate on Carleson sets on the unit circle. We also conjecture that the equivalence (ii) $\Leftrightarrow$ (iii) holds even when $0<\alpha<1$, but we have been unable to verify this.

Acknowledgments. The authors deeply thank the referee for many valuable remarks and suggestions.

This work was supported by the Fonds Racine.

\section{References}

[B] K. Bogdan, On the zeros of functions with finite Dirichlet integral, Kodai Math. J. 19 (1996), 7-16.

[Car1] L. Carleson, Sets of uniqueness for functions regular in the unit circle, Acta Math. 87 (1952), 325-345.

[Car2] - On the zeros of functions with bounded Dirichlet integrals, Math. Z. 56 (1952), 289-295.

[Car3] - A representation formula for the Dirichlet integral, ibid. 73 (1960), 190-196.

[Cau1] J. G. Caughran, Two results concerning the zeros of functions with finite Dirichlet integral, Canad. J. Math. 21 (1969), 312-316. 
[Cau2] J. G. Caughran, Zeros of analytic functions with infinitely differentiable boundary values, Proc. Amer. Math. Soc. 24 (1970), 700-704.

[M] J. Mashreghi, Representation Theorems in Hardy Spaces, London Math. Soc. Student Texts 74, Cambridge Univ. Press, 2009.

[MS] J. Mashreghi and M. Shabankhah, Zero sets and uniqueness sets with one cluster point for the Dirichlet space, J. Math. Anal. Appl. 357 (2009), 498-503.

[NRS] A. Nagel, W. Rudin and J. H. Shapiro, Tangential boundary behavior of functions in Dirichlet-type spaces, Ann. of Math. (2) 116 (1982), 331-360.

[Ne] J. D. Nelson, A characterization of zero sets for $\mathcal{A}^{\infty}$, Michigan Math. J. 18 (1971), 141-147.

[No] W. P. Novinger, Holomorphic functions with infinitely differentiable boundary values, Illinois J. Math. 15 (1971), 80-90.

[RRS] S. Richter, W. T. Ross and C. Sundberg, Zeros of functions with finite Dirichlet integrals, Proc. Amer. Math. Soc. 132 (2004), 2361-2365.

[SS] H. S. Shapiro and A. L. Shields, On the zeros of functions with finite Dirichlet integral and some related function spaces, Math. Z. 80 (1962), 217-229.

[TW] B. A. Taylor and D. L. Williams, Zeros of Lipschitz functions analytic in the unit disc, Michigan Math. J. 18 (1971), 129-139.

Javad Mashreghi

Mahmood Shabankhah

Département de mathématiques

CMI, LATP

et de statistique

Université de Provence

Université Laval

Québec, QC, Canada G1K 7P4

39, Rue F. Joliot-Curie

E-mail: javad.mashreghi@mat.ulaval.ca E-mail: mahmood.shabankhah@cmi.univ-mrs.fr

Received May 27, 2009

Revised version January 7, 2010

(6636) 